\title{
SOROPREVALÊNCIA E FATORES DE RISCO PARA SÍFILIS EM POPULAÇÃO CARCERÁRIA DE GOIÁS(1)
}

Ana Lúcia Sampaio Sgambatti de ANDRADE (2), Celina Maria Turchi MARTELLI (2), Luiz Carlos Silva SoUSA (3), Marta Antunes de SOUSA (4) \& Fabio ZICKER (2).

\section{RESUMO}

Com o objetivo de dimensionar a prevalência da infecção pelo Treponema pallidum e determinar fatores de risco relacionados a soropositividade foram rastreados 299 presidiários no Centro Penitenciário de Atividades Industriais de Goiás (CEPAIGO), $20 \mathrm{Km}$ de Goiânia. O rastreamento sorológico foi realizado utilizando-se como critério de positividade, qualquer resultado sororeagente ao VDRL independentemente do título. Através de um questionário padronizado foram avaliados os seguintes fatores de risco: tempo de encarceramento, sinais e sintomas relativos às principais doenças sexualmente transmissíveis (DST), história de sífilis ou outras DST e práticas sexuais (homo/bissexualismo e número de parceiros). Foram calculados o valor preditivo positivo (VPP) e negativo (VPN) da história pregressa de sífilis obtida na anamnese. Uma soroprevalência global de $18,4 \%$ foi obtida, não havendo diferença entre as faixas etárias. O VPP do antecedente de sífilis foi de $26 \%$ signifi cando que $74 \%$ dos individuos que referiram sífilis no passado não tiveram confirmação pelo VDRL. Entre os fatores de risco testados, a bissexualidade foi o único que apresentou associação estatisticamente significante com soropositividade (risco relativo 5,8 - LC $95 \% 1,2-16,0 \mathrm{p}=0,03$ ). Foram discutidas as dificuldades metodológicas que poderiam ter influenciado nos resultados.

UNITERMOS: Rastreamento sorológico; Soroprevalência; Sifilis; Fatores de risco; Doenças sexualmente transmissiveis.

\section{INTRODUÇÃO}

A epidemia da Síndrome de Imunodeficiência Adquirida (AIDS/SIDA) trouxe como conseqüência um aumento da freqüência de diagnóstico das doenças sexualmente transmissiveis, principalmente em homossexuais. Este fato tem contribuido para um crescente número de inves- tigaçôes em populaçōes consideradas de risco para as doenças sexualmente transmissiveis, na tentativa de identificar co-fatores relacionados ao Vírus da Imunodeficiência Humana (VIH). A similaridade das características de transmissão deste grupo de doenças, especialmente a sífilis

(1) Trabalho financiado pela secretaria de Ciência e Tecnologia do Ministério da Saúde e CNPq.

(2) Departamento de Saúde Coletiva do Instituto de Patologia Tropical e Saúde Pública da Universidade Federal de Goiảs. Goiânia, Goiás, Brasil

(3) Hospital de Doenças Tropicais de Goiania - Secretaria Estadual de Saúde (SUDS).

(4) Bolsista do CNPq

Endereço para correspondência: Dra. Ana Lúcia S. S. de Andrade. Rua 1136, n: 630 - Setor Marista. CEP 74000 Goiânia Goiás, Brasil. 
ANIDRADF. A. L. S. S. de: MARTELLI, C. M. T.; SOUSA. L. C. S. SOUSA. M. A. de \& ZICKER. F. - Soroprevalencia " falores de risco para sifilis em populacácarceraria de Goias. Rev. Inst. Med. trop. S. Paulo, 31 (3). 177 182. 1989

e hepatite $B$, justificam o aprofundamento dos estudos epidemiológicos nas doenças sexual mente transmissiveis.

A falta de registros oficia is sobre a incidência de sifilis em nosso meio, fazem com que dados de soroprevalência sejam usualmente obtidos em 3 situaçōes distintas: 1) em rastreamentos sorologicos em bancos de sangue ${ }^{1.18} ; 2$ ) em ambulatórios de pré-natal ${ }^{3 .}$, dermatologia e doenças sexuaimente transmissiveis ${ }^{17}$ e 3 ) em inquéritos especiais com finalidade de pesquisa, principal mente em grupos considerados de risco ${ }^{2.8 .16}$

Poucos são os estudos realizados em populaçōes sob regime de reclusão ${ }^{2}$. 6. 8. 19 . Altas taxas de soropositividade para sífilis tem sido registradas nestes grupos populacionais, mas pouco tem sido discutido sobre os aspectos epidemiológicos relacionados à infecçāo pelo T. pallidum. A organização social, bem como o estilo de vida destes individuos, fazem deste "grupo social", uma população de risco para as doenças sexualmente transmissiveis. A prática homossexual é comum $^{13}$, relacionada talvez com o tempo de en carceramento. Este poderia ser um fator condicionante do risco, uma vez que o regime de reclusăo poderia motivar alterações nas práticas se xuais.

Neste sentido, o objetivo deste estudo foi de dimensionar a prevalència da infecçāo pelo $\mathbf{T}$. pallidum na população prisional de Goiás e ava liar fatores de risco a ela relacionados. Este estu do pretende oferecer subsídios para a implan taçāo de medidas de saúde pública, visando o controle da infecçăo.

\section{MATERIAL E MÉTODOS}

\section{População de estudo}

$O$ estudo foi realizado durante os meses de junho e julho de 1988, no Centro Penitenciário de Atividades Industriais de Goiás (CEPAIGO), municipio de Aparecida, $20 \mathrm{~km}$ de Goiânia. A população de 301 homens detentos, encontra va-se distribuída em 2 situaçōes carcerárias: intramuros (210-69,8\%), os detidos em celas individuais, e, extramuros $(91-30,2 \%)$, os residentes em agrovilas nas vizinhanças do presidio. Apenas ¿detentos recusaram-se a participar.
O projeto foi submetido a apreciação e aprovado junto a Secretaria da Justiça, Secretaria da Saúde e pelo Conselho Coordenador de Ensino e Pesquisa da UFG.

\section{Exame sorológico}

Foram coletados de cada indivíduo aproximadamente $10 \mathrm{ml}$ de sangue pelo sistema vacutainer ${ }^{\circledR}$, para a realizaçāo do teste de VDRL (Venereal Disease Research Laboratories Tests $)^{10}$, preenchendo-se simultaneamente uma ficha de identificação sumária.

Os testes foram realizados no laboratório do Hemocentro de Goiás (SUDS) de acordo com a técnica descrita por HARRIS e cols ${ }^{10}$, utilizan do-se kits Laborclin. Foram considerados como testes positivos para efeito de rastreamento todos os resultados reagentes ao VDRL independentemente do título. Resultados positivos em titulaçōes maiores ou iguais a 1:4 foram orientados para tratamento.

\section{Questionário}

Após o rastreamento sorológico todos indi viduos foram solicitados a comparecer ao servi ço médico do presídio para uma investigaçāo clínica-epidemiológica. Um único entrevistador treinado (médico especialista em doenças infecciosas), desconhecendo o resultado do exame sorológico, aplicou um questionário padronizado, colhendo informaçōes sobre identificação, procedència (rural/urbana), idade, ocupaçāo, estado civil, tempo de encarceramento, sinais e sintomas relativos as principais doenças sexualmen. te transmissiveis, história de sifilis ou outras DST (hepatite B, AIDS, gonorréia, herpes, condiloma, linfogranuloma e cancróidel e práticas sexuais (homo/bissexualismo e número de parceiros no último ano).

A procedência rural/urbana foi definida em relação ao local de moradia anterior à prisão. O tempo de encarceramento foi calculado como o somatório dos anos (completos) de reclusāo para cada indivíduo.

A entrevista só pode ser realizada em 201 $(67.2 \%)$ individuos testados sorologicamente, por dificuldades de acesso aos detentos. 
ANDRADE, A. L. S. S. de; MARTELL1. C. M. T.: SOUSA. L. C. S.: SOUSA. M. A. de \& ZICKER. F. - Soroprevalencia e fatores de risco para sifilis em populaça carceraria de Goias. Kev. Inst. Med. trop. S. Paulo, 31(3): 177 182. 1989.

\section{Análise estatistica}

Os dados da ficha de identificaçáo, questionário e resultados sorológicos foram digitados em microcomputador utilizando-se o programa "DBASE III +" e foram analisados através do programa "EPI INFO - Questionnaire processing program" (Centers for Disease Control, Atlanta, USA, 1987).

Foram calculadas taxas de soroprevalencia por faixa etária e os riscos relativos ("odds ratio") associados ao tempo de encarceramento, procedencia (rural versus urbana) e "comportamento em risco" (história de DST, de sifilis, preferencia sexual e número de parceirosı em relação à soro positividade ao VDRL. Testes de significância e limites de confiança de $95 \%$ (Woolf) foram esti. mados.

Foi calculado o valor preditivo positivo (VPP) e negativo (VPN) da historia pregressa de sifilis colhida na entrevista, ou seja, a probabilidade de se obter exame sorológico positivo em individuos que relataram história de sifilis (VPP), ou exame negativo na auséncia de tal história (VPN).

\section{RESULTADOS}

\section{Soroprevalência}

A média de idade da populaçāo de detentos foi de 31,6 anos $(+/-9,1)$. O rastreamento sorológico para sifilis detectou uma soroprevalência de $18,4 \%$ (55 individuos). Alterando-se o ponto de corte da positividade para um título igual ou superior a 1:4, aumentando assim a especificidade do VDRL, a soroprevalência diminuiu para $6,7 \%$ (20 positivos).

A tabela 1 mostra a soroprevalência por faixa etária na população carcerária, observandose valores semelhantes nas faixas etárias de 18 a 29,30 a 39 e 40 a 49 anos. Foi observada uma variação da soroprevalência de $6 \%$ a $9 \%$, em todas as faixas etárias, quando adotou-se como soro positivo resultados do VDRL com título maior ou igual a $1: 4$.

\section{Fatores de risco}

Foram investigados clínica-epidemiologicamente $41(74,5 \%)$ dos 55 detentos soropositivos
TABELA 1

Soroprevaléncia para sifilis por faixa etária na população car ceráría do CEPAIGO, Goiás, 1988.

\begin{tabular}{lrc}
\hline Faixa etária & Populaçảo & $\begin{array}{c}\text { Prevaléncia } \\
(\%)\end{array}$ \\
\hline & & \\
$18-29$ & 141 & 19,1 \\
$30-39$ & 107 & 18,7 \\
$40-49$ & 34 & 20,5 \\
$>50$ & 17 & 11,7 \\
\hline TOTAL & 299 & 18,4 \\
\hline
\end{tabular}

e 160 (65,6\%) dos 244 soronegativos. A perda de casos deveu-se a dificuldades no recrutamento desses indivíduos por motivo de liberdade condicional, transferência e fuga.

A tabela 2 apresenta o risco relativo, limites de confiança $95 \%$ e teste de significância, associado às variáveis investigadas. $3.5 \%$ (7) dos detentos entrevistados referiram práticas bisse xuais, sendo este o único fator que apresentou asśociaçāo estatisticamente significante com soropositividade.

A história de residència, colhida detalhada mente, permtiu verificar a freqüente migração destes individuos, dificultando por vezes, carac. terizar de forma adequada, a procedência rural e a urbana Cerca de $76 \%$ da população carce. rária foi registrada como procedente de zona urbana, principalmente de municípios do interior do estado.

O valor preditivo positivo e negativo da história pregressa de sifilis foi de $26 \%$ e $80 \%$ respectivamente, conforme dados da tabela 3 .

\section{DISCUSSÃO}

O presente estudo foi delineado para dimen sionar a prevalência da infecçāo pelo T. pallidum, através de rastreamento sorológico, em po pulação considerada de risco para doenças se xualmente transmissiveis. Nesta situaçāo o VDRL tem sido o teste preconizado por ser de fácil execuçāo e baixo custo, embora nảo seja específico para sífilis (teste nāo treponêmico) ${ }^{12}$ 15 . 
ANDRADF, A. L. S. S. de: MARTELLI, C. M. T.; SOUSA, L. C. S.; SOUSA. M. A. de \& ZICKER F. - Soroprevaléncia e latores de risco para sifilis em populaçao carceraria de Goias. Rev. Inst. Med. trop. S. Paulo, $31(3)$ : 177 182, 1989.

TABELA 2

Fatores de risco associados a sífilis em população carcerária, CEPAIGO — Goiás, 1988.

\begin{tabular}{|c|c|c|c|c|c|}
\hline Fatores de risco & População & $\begin{array}{l}\text { Soropreva- } \\
\text { lencia }(\%)\end{array}$ & $\begin{array}{l}\text { Risco } \\
\text { relativo }\end{array}$ & $\begin{array}{c}\text { Limites de } \\
\text { confiança } 95 \%\end{array}$ & Valor de $p$ \\
\hline \multicolumn{6}{|l|}{ Procedencia } \\
\hline Rural & 47 & 14,9 & 1,0 & & \\
\hline Urbana & 154 & 22,1 & 1,6 & $(0,62-4,36)$ & $>0,05$ \\
\hline \multicolumn{6}{|c|}{ Anos de Encarceramento } \\
\hline$<1$ & 31 & 19,4 & & & $>0,05$ \\
\hline $1-2$ & 102 & 18,6 & & & $\mathrm{X}_{3}^{2}=0,68$ \\
\hline $3-4$ & 27 & 22,2 & & & \\
\hline$>5$ & 41 & 24,4 & & & \\
\hline \multicolumn{6}{|l|}{ IIistória de DST } \\
\hline Nào & 77 & 22,1 & 1,0 & & \\
\hline Sim & 124 & 19,4 & 0,85 & $(0,4-1,81)$ & $>0,05$ \\
\hline \multicolumn{6}{|l|}{ Historia de Sifilis } \\
\hline Nāo & 178 & 20,2 & 1,0 & & \\
\hline $\operatorname{sim}$ & 23 & 26,1 & 1,5 & $(0,5-4,27)$ & $>0,05$ \\
\hline \multicolumn{6}{|l|}{ Atividade Sexual } \\
\hline Heterossexual & 192 & 18,8 & 1,0 & & \\
\hline Bissexual & 7 & 57,1 & 5,78 & $(1,2-26)$ & 0,03 \\
\hline \multicolumn{6}{|c|}{ N: de Parceiros no último ano } \\
\hline 0 & 39 & 20,5 & 1,0 & & \\
\hline 1 & 111 & 19,8 & 0,96 & $(0,36-2,62)$ & $>0,05$ \\
\hline$>1$ & 51 & 21,6 & 1,07 & $(0,34-3,34)$ & $>0,05$ \\
\hline
\end{tabular}

População total $=201$

Prevalència $=20 \%$

TABELA 3

Valor preditivo positivo (VPP) e negativo (VPN) da história pregressa de sifilis lanamnese). Populaça carccrária, CEPAI GO, Goiás, 1988.

\begin{tabular}{lcccc}
\hline & \multicolumn{2}{c}{ VDRL } & Total \\
& POS & NEG & \\
\hline $\begin{array}{l}\text { Historia } \\
\text { de } \\
\text { Sifilis }\end{array}$ & Sim & 6 & 17 & 23 \\
\hline Tótal & Nào & 35 & 143 & 178 \\
\hline
\end{tabular}

VPP $6.23=26 \%$

VPN $143.178=80 \%$

Considerando se que rastreamentos sorológicos tem como objetivo a detecçāo de infecção atual ou pregressa. a utilizaçào da positividade ao VDRL em qualquer título, permite identificar o maior número de indivíduos com contacto previo com o T.pallidum. Por outrc lado, esta estratégia leva a obtençào de resultados falsos positivos em certa proporçâo, nāo só pela inespecificidade do teste, como também pelo ponto de corte utilizado ${ }^{15}$.

A positividade no VDRL de $18.4 \%$ encon trada, confirma a alta prevalência de sifilis neste grupo social. Uma prevalencia de positividade ao VDRL de $4 \%$ foi obtida recentemente em nosso meio, utilizando-se o mesmo ponto de corte, em 62814 candidatos a doaçào de sangue, população predominantemente do sexo mascuino e maior de 18 anos $^{1}$. Igualmente, outros estudos em reclusos, tem mostado altas taxas de prevaléncia para sifilis, variando de $14 \%$ a $25 \%^{2.8}$. 
ANDrade, A. L. S. S. de; MARTELLI, C. M. T.; SOUSA, L. C. S.: SOUSA, M. A. de \& ZICKER. F. - Soroprevaléneia e fatores de risco para sifilis em populaçāo carcerária de Goiás. Rev. Inst. Med. trop. S. Paulo, 31 (3): 177 182, 1989.

A análise da prevalência por faixa etária mostrou uma distribuição homogénea nas diferentes décadas e talvez por tratar-se de uma populaçāo jovem $180 \%$ de individuos com idade in ferior a 40 anos), nāo foi possivel detectar nenhu ma relaçāo da soropositividade com idade.

A comparação entre as prevalências segun do procedência rural e urbana não apresentou diferença estatisticamente significante. Talvez a caracterizaçāo da procedência em capital e in terior fosse mais adequada para espelhar os aglomerados populacionais, com possiveis diferen ças quanto ao estilo de vida e diferentes riscos de infecçâo.

Antecedentes de DST e de sífilis não mostraram associaçāo estatisticamente significante com soropositividade. O baixo VPP $(26 \%)$ do an tecedente de sifilis, significa que $74 \%$ dos indiví duos que referiram a doença no passado, näo tiveram confirmação pelo VDRL. Como o VPP depende da prevalência da doença em estudo, seria razoável esperar valores maiores ${ }^{9}$. Vários fatores poderiam explicar o baixo rendimento da anamnese como fator de predição da sorologia. A falta de informaçāo sobre doenças venéreas, a dificuldade em distinguir a sifilis das demais infecçōes poderiam tanto subestimar como su perestimar a sua ocorréncia. Por outro lado, como não foi determinada a época da doença no passado poderia ter ocorrido uma perda do mar cador sorológico explicando assim a nāo corres pondência do resultado sorológico com a informaçāo colhida na anamnese ${ }^{14}$

O comportamento sexual, avaliado pelo número de parceiros e homossexualidade, tem sido apontado como fator de risco para sífilis ${ }^{4}$. Mesmo considerando as dificuldades inerentes a obten ção de respostas confiáveis em relação a estas variáveis, $3.5 \%$ dos entrevistados relataram práticas bissexuais. Neste estudo, a bissexualidade foi o único fator estatisticamente significante associado a soropositividade, obtendo-se um risco relativo de 5,78 (limites de confiança $95 \%, 1,2-26$ ).

O tempo de encarceramento foi avaliado como fator de risco para a infecçāo entendendo-se que esta condiçāo seria favorável ao homosse xualismo. No entanto, não encontramos uma as sociação positiva com relaçāo a esta variável.
A forma de coleta desta informação, não compu tando anos contínuos de cárcere e sim o soma tório dos anos (completos) de reclusão de cada indivíduo, pode ter interferido nesta avaliação A associação entre encarceramento e homo bis sexualidade, poderia ser verificada comparando-se a freqüencia deste comportamento na populaçāo em geral e em individuos em regime de reclusão, através de estudos populacionais. Es tas investigaçōes seriam necessárias para a defi nição de estratégias de saúde pública para o con trole das doenças sexualmente transmissiveis.

\section{SUMMARY}

\section{Seroprevalence and risk factors for syphilis in prisoners in Goiás, Brazil.}

A cross-sectional survey was carried-out among 299 prisoners in the Penitentiary Center of Industrial Activity of Goiás (CEPAIGO), to determine the seroprevalence to $T$. pallidum and to identify risk factors associated to seropo sitivity. The seropositivity criterion was a positive VDRL test at any titer. A questionnaire was applied to evaluate the following risk factors: time of imprisonment, clinical evidence of sexual. ly transmitted diseases (STD), history of syphilis or others STD, homo/bisexuality and number of sexual partners. The positive (PPV) and negative (NPV) predictive values of the history of sy philis were calculated. Seroprevalence of $18,4 \%$ was found and no difference was detected in the different age groups. The PPV of history of syphilis was $26 \%$ indicating that $74 \%$ of the indivi duals who have reported syphilis in the past pre sented a negative VDRL test. Among all the risk factors studied, homo/bisexuality was the only one with statistically significant association with seropositivity (relative risks $5.7-95 \%$ CL1.2-26, $p=0.03$ ). The paper discusses the me thodological problems related with the investi gation.

\section{REFERÊNCIAS BIBLIOGRÁFICAS}

1. ANDRADE, A. L. S. S ; MARTELLI, C. M. T. \& ZICKER F. - Rastreamento sorológico em banco de sangue como indicador de morbidade populacional Rev. Salide públ S. Paulo, 23: $20 \cdot 25,1989$

2. AZULAY, R. D.; RICAR'T, J. C. C.; MONTEIRO, C. A A.; GRIPP, A. C.; RODRIGUES, P. C.; ZANON, U. \& GA 
ANDRADE, A. L. S. S. de; MARTELLI, C. M. T.; SOUSA, L. C. S. SOUSA, M. A. de \& ZICKER, F. - Soroprevalencia e fatores de risco para sifilis em populaçāo carcerária de Goias. Rev. Inst. Med. trop. S. Paulo, 31 (3): 177 182, 1989.

MEIRO, N. F. - Sífilis: inquérito sorológico em diferentes grupos sócio-econômicos na cidade de Niterói. Rev. Ass. méd. bras., $25: 85-86,1979$.

3. CUNHA, V. F ; CARVALHO, J. P. P. \& BELDA, W. Aspectos do problema da sifilis em duas unidades de aten. ciào a gestantes na capital de Sāo Paulo. An. bras Derm., 58: 6366,1983 .

4. DARROW, W. W.; BARRETT, D.: JAY, K. \& YOUNG, A. - The gay report on sexually transmitted diseases. Amer. J. publ. Hith., 71: 1004-1011, 1981

5. DOLNIKOFF, M. FARAH, A. C. KUPERMAN, J. L. \& DELASCIO, D. - Incidência da sifilis na gravidez. J. bras. Ginec., 81 : 261,1976

6. GOLDENBERG, P.; VAISMAN, E.; PORTO, J. A. de SBRISSA, E.: NAGY, M. \& LESER, W. P. - Coeficientes de positividade das reaçōes de Wasserman e VDRL em convocados para Serviço Militar no estado de Sảo Paulo de 1968 a 1971. Rev. Ass. méd. bras., 20: 265, 1974.

7. GOLDENBERG, P.; PARDO, R. M.; NETO, D. R.; RIBEIRO, S. A.; PERNAMBUCO, M. \& YUNES, M. - A dimensāo social das doenças. II - Positividade das reaçós de Wasserman e VDRL em convocados para o Serviço Militar no Estado de São Paulo (Brasil), de 1972 a 1978. Rev. Saúde púbI. (S. Paulo), 16: 133-143, 1982.

8. GOMES, S. - Incidencia de reaçoes sorológicas positivas para sifilis na populaçāo carcerảria de Niterói. Arq. bras.

v Med., 59: 275 278. 1985

9. GOODHART, G. L. - Use and interpretation of serologic tests for the diagnosis of syphilis. Sth. med. J. (Bgham, Ala.), 76: $373-379,1983$

10. HARRIS, A.: ROSENBERG, A. A. \& RIEDEL, L. M. A microflocculation test for syphilis using cardiolipin anti. gen. Preliminary report. J. vener. Dis. Inform., 27: 169-174. 1946
11. JUDSON, F N.: PENLEY, K. A.: ROBINSON, M. E. \& SMITH, J. K. - Comparative prevalence rates of sexually transmitted diseases in heterosexual and homosexual men. Amer. J. Epidem., 112: 836-843, 1980.

12. LEE, J. T. \& SPARLING, F. -- Syphilis. An algorithm J. Amer. med. Ass., 242: 1187-1189, 1979.

13. MINISTERIO DA SAÚdE, DIVISÁO NACIONAL DE CONTROLE DE DOENÇAS SEXUALMENTE TRANS MISSIVEIS - SIDA/AIDS \& MINISTERIO DA JUSTI CA, DEPARTAMENTO PENITENCIARIO - Recomen daçóes preliminares para controle da infecçào pelo HIV em presidiários. Brasília, 1987

14. MOORE, J.E. \& MOHR, C. F. - Biologically false positive serologic tests for syphilis. Type, incidence, and cause. J. Amer. med. Ass., 150: 467-473, 1952

15. OATES, J. K. - Serological tests for syphilis and their clinical use. Brit. J. Hosp. Med., 13: 612-617, 1979

16. PEREIRA M G - Inquérito sorológico de sífilis em adultos: Planaltina, DF, Brasil, 1977. Rev. Saúde públ. (S. Paulo), 14: $358 \cdot 365,1980$

17. SITTART, J.A.; TAYAH, M.\& SOARES, Z. - Incidência da sífilis no serviço de dermatologia do Hospital do Servidor Público do Estado de Sào Paulo. Rev. paul. Med., 101: $29-30,1983$

18. TAKEDA, A. K : NAKAMURA, P M \& BARBOSA, F. C. - Estudo comparativo entre as reaçóes de floculação, fixaçāo de complemento, imunofluorescência indireta e micro-hemaglutinação passiva para sífilis. Rev. Inst. Adolfo Lutz, 39: 137-144, 1979.

19. THIN, R. N. \& O'RORKE. C. M. - Results of serological test for syphilis among Gurkhas and other high risk groups. Genitourinary Med., 61: 33-35, 1985.

Recebido para publicação em 17/11/1988 